\title{
Continuous Flow Supercritical Water Synthesis and Temperature Dependent Defect Structure Analysis of YAG and YbAG Nanoparticles
}

Peter Nørby ${ }^{1}$, Kirsten M. Ø. Jensen ${ }^{2}$, Nina Lock ${ }^{3}$, Mogens Christensen ${ }^{1}$, Bo B. Iversen ${ }^{1} *$

${ }^{1}$ Center for Materials Crystallography, Department of Chemistry and iNANO, Langelandsgade 140, DK-8000 Aarhus C, Denmark

${ }^{2}$ Dept. of Chemistry, University of Copenhagen, Universitetsparken 5, DK-2100 København Ø, Denmark

${ }^{3}$ Dept. of Chemistry and iNANO, Gustav Wieds Vej 14, DK-8000 Aarhus C, Denmark

*Corresponding author: bo@chem.au.dk 


\section{The garnet structure}

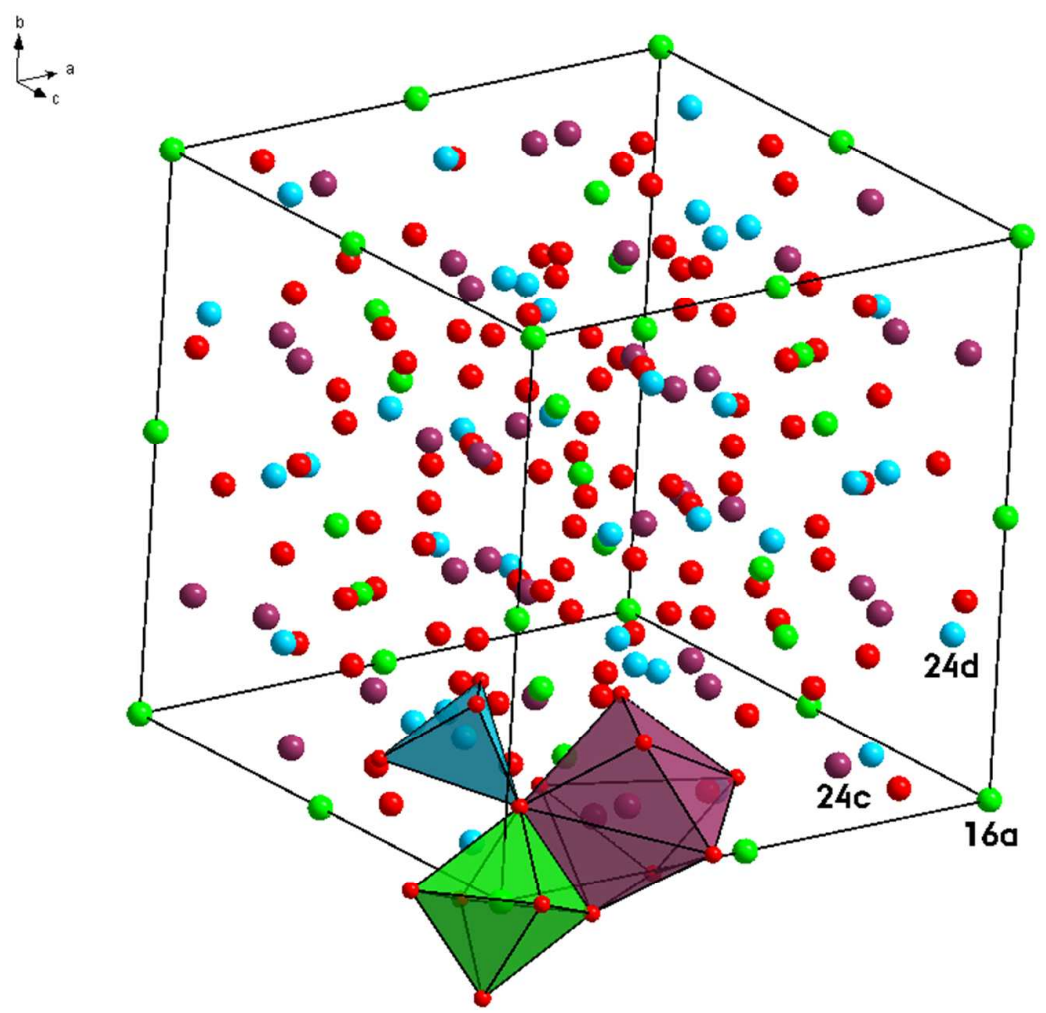

Figure S1: The unit cell with the three different sites coordination environments shown. The Wyckoff sites are given in the table.

\begin{tabular}{|c|c|c|}
\hline Atom & Wyckoff site & Color \\
\hline $\mathrm{Y} / \mathrm{Yb}$ & $24 \mathrm{c}$ & Purple \\
\hline $\mathrm{Al}$ & $16 \mathrm{a}$ & Green \\
\hline $\mathrm{Al}$ & $24 \mathrm{~d}$ & Blue \\
\hline $\mathrm{O}$ & $96 \mathrm{~h}$ & Red \\
\hline
\end{tabular}




\section{Schematic of the continuous flow reactor}

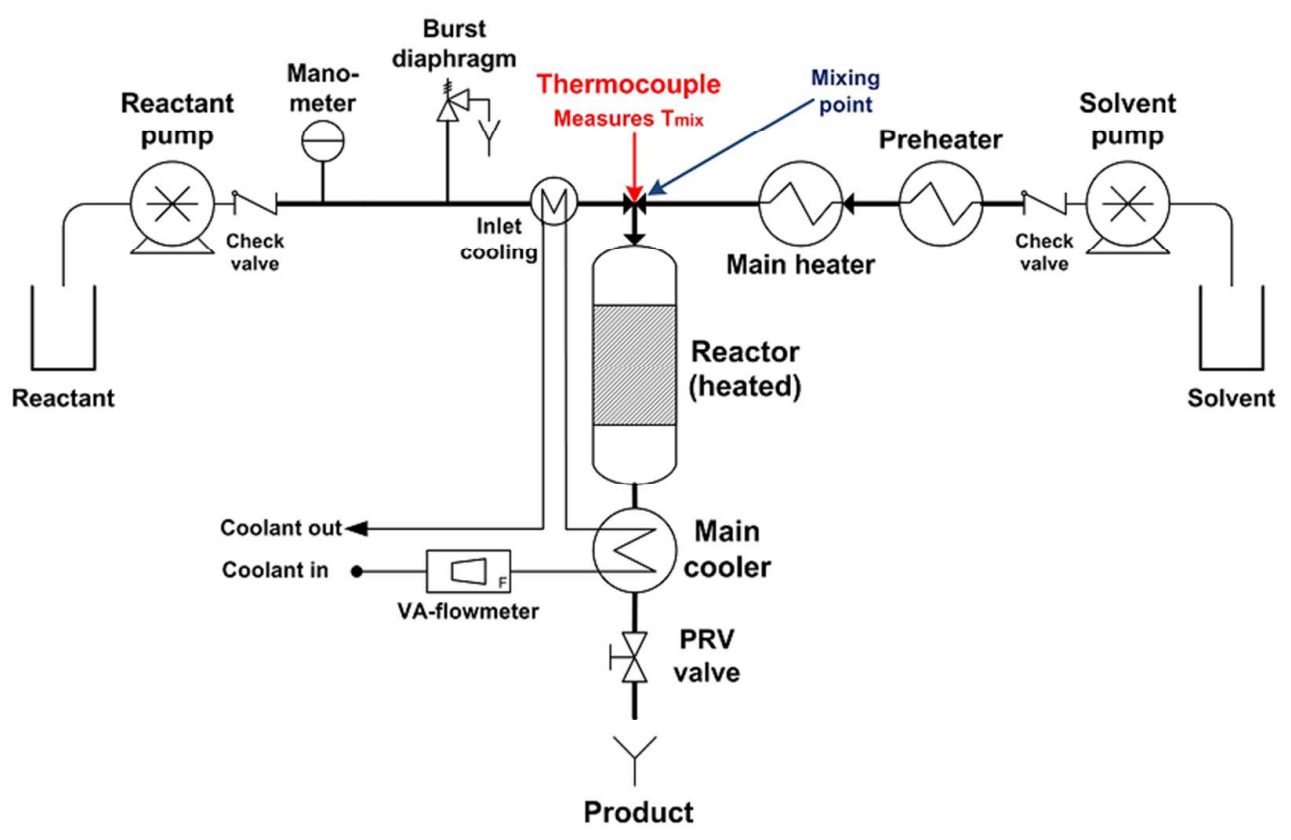

Figure S2: Illustration of the continuous flow reactor.

The synthesis procedure can be divided into five steps: 1) Cleaning the entire pressurized system with deionised water. 2) Reaching the desirable temperatures for the preheater, solvent heater, and reaction chamber. 3) When a steady-state was reached, the reaction was initiated by pumping one of the precursors into the reactor (instead of solvent) using the reactant pump. 4) Collection of a milky white/yellow solution at the product outlet. The first part of the product was discarded as it might contain impurities, so the actual product was collected after approximately $20 \mathrm{~s}$. 5) The product was dried at room temperature. 
Supporting information

\section{TEM pictures}

The TEM pictures shown in the figure below shows that the morphology and size of the nanoparticles are independent of synthesis temperature used in the flow set-up

(a)

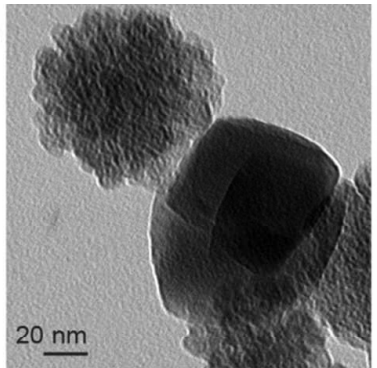

(d)

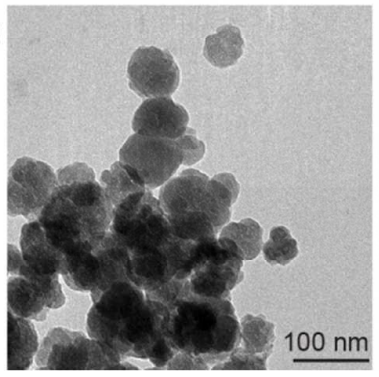

(b)

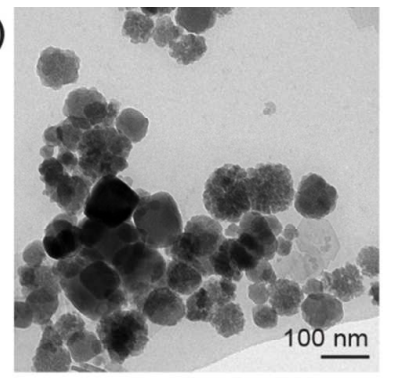

(e)

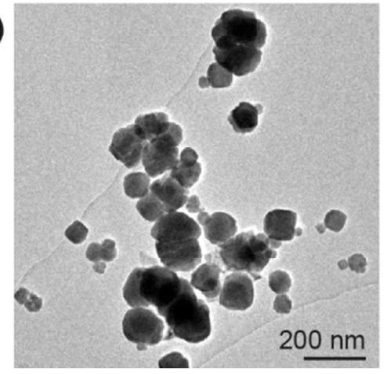

(c)

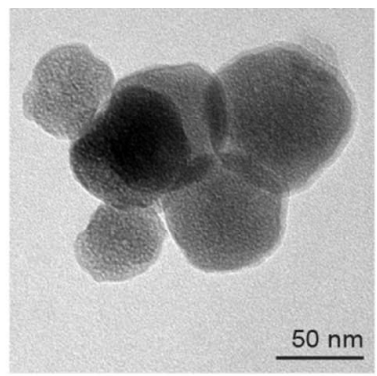

(f)

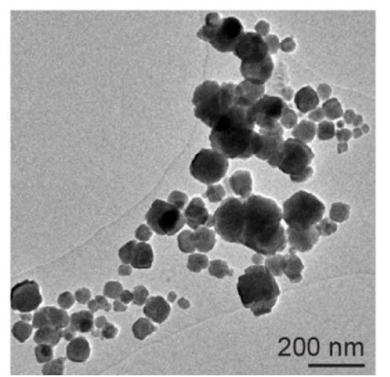

Figure S3. TEM pictures of the as-synthesized nanoparticles of $\mathrm{YAG}$ and $\mathrm{YbAG}$. (a) YAG, $300{ }^{\circ} \mathrm{C}$, (b) $\mathrm{YAG}, 350{ }^{\circ} \mathrm{C}$, (c) $\mathrm{YAG}, 400{ }^{\circ} \mathrm{C}$, (d) $\mathrm{YAG}, 450{ }^{\circ} \mathrm{C}$, (e) $\mathrm{YbAG}, 400{ }^{\circ} \mathrm{C}$, (f) $\mathrm{YbAG}, 450{ }^{\circ} \mathrm{C}$. 
Overview TEM pictures of $\mathrm{YbAG}, 400{ }^{\circ} \mathrm{C}$
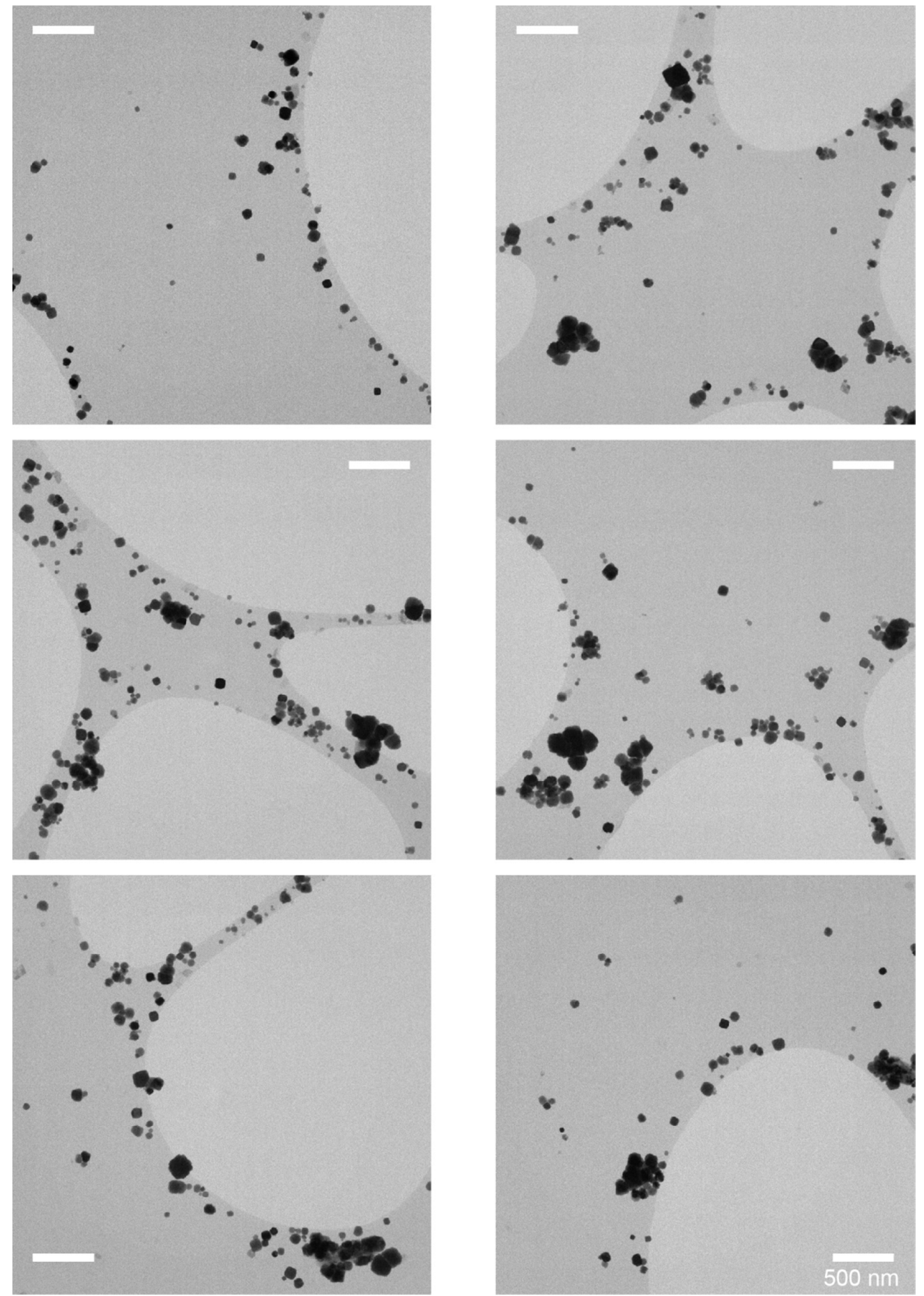

Figure S4: The nanoparticle size distribution is homogenous and the morphology is similar for the whole sample. The scale bar is the same in all figures. 
Overview TEM pictures of YAG, $400{ }^{\circ} \mathrm{C}$
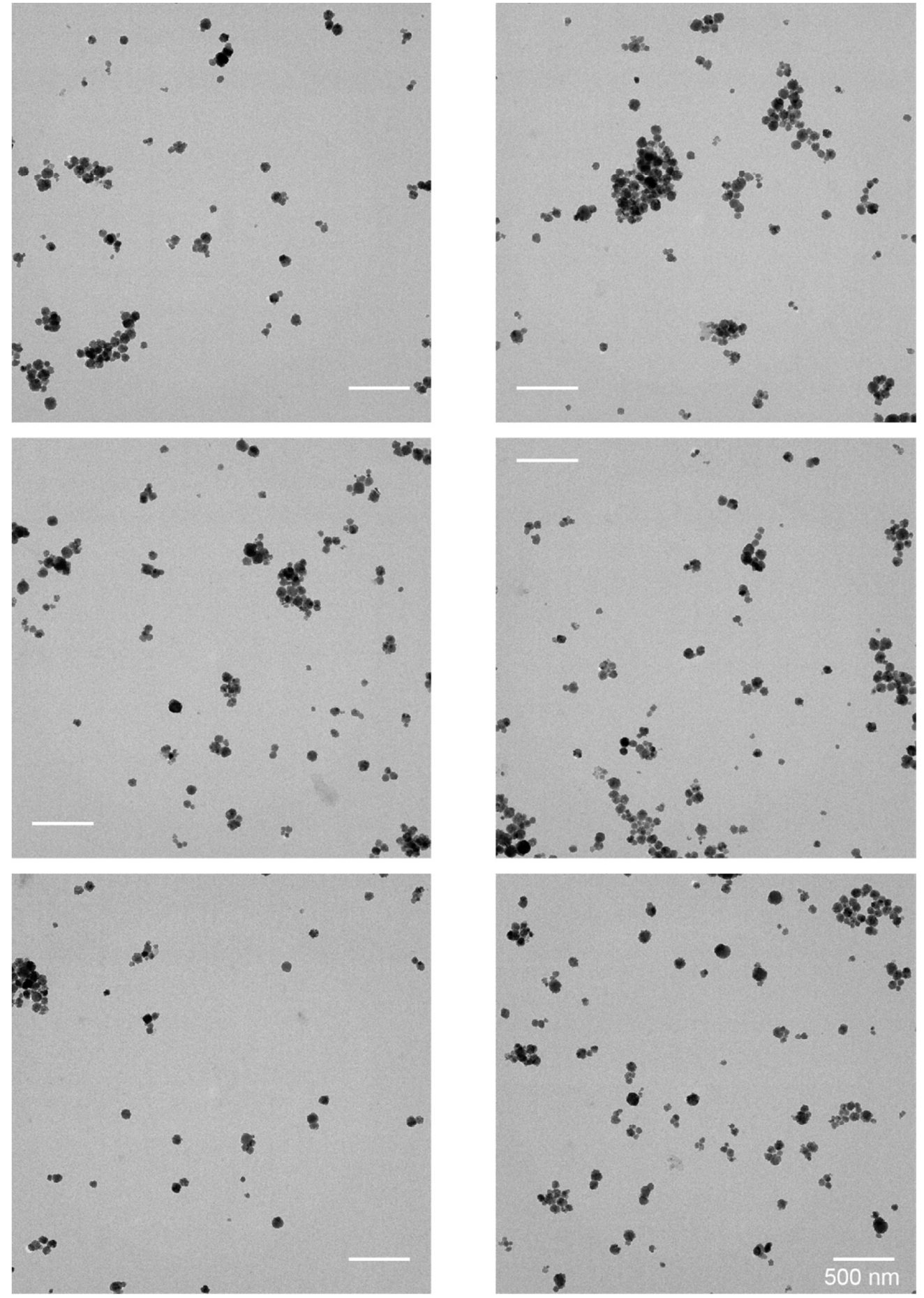

Figure S5: The nanoparticle size distribution is homogenous and the morphology is similar for the whole sample. The scale bar is the same in all figures. 
EDS mapping of YAG nanoparticles synthesized at $400{ }^{\circ} \mathrm{C}$
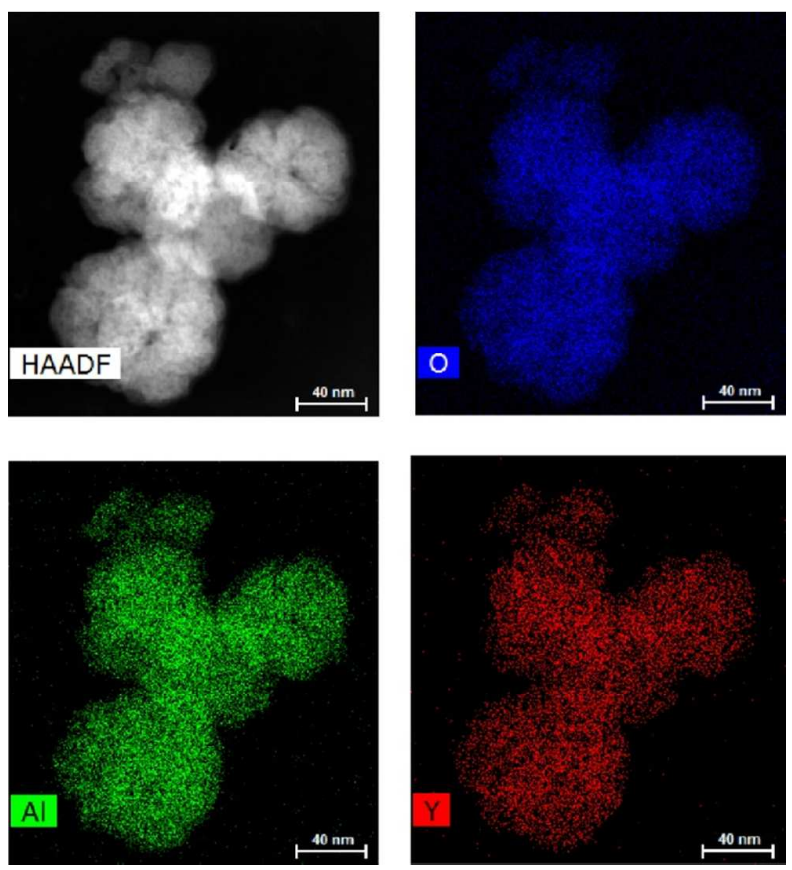

Figure S6: HAADF STEM image of YAG nanoparticles synthesized at $400{ }^{\circ} \mathrm{C}$ and elemental mapping.

\section{Measurement order of PXRD data}

Graphic illustration of the measurement order at SPring-8 of the YAG and YbAG samples synthesized at $400^{\circ} \mathrm{C}$. Two different set-ups have been used in the temperature range, a low and high temperature set-up. A small overlap between the two set-ups is used in order to see if the crystallographic structure has changed during the change of set-up and as a check of the temperature calibration.
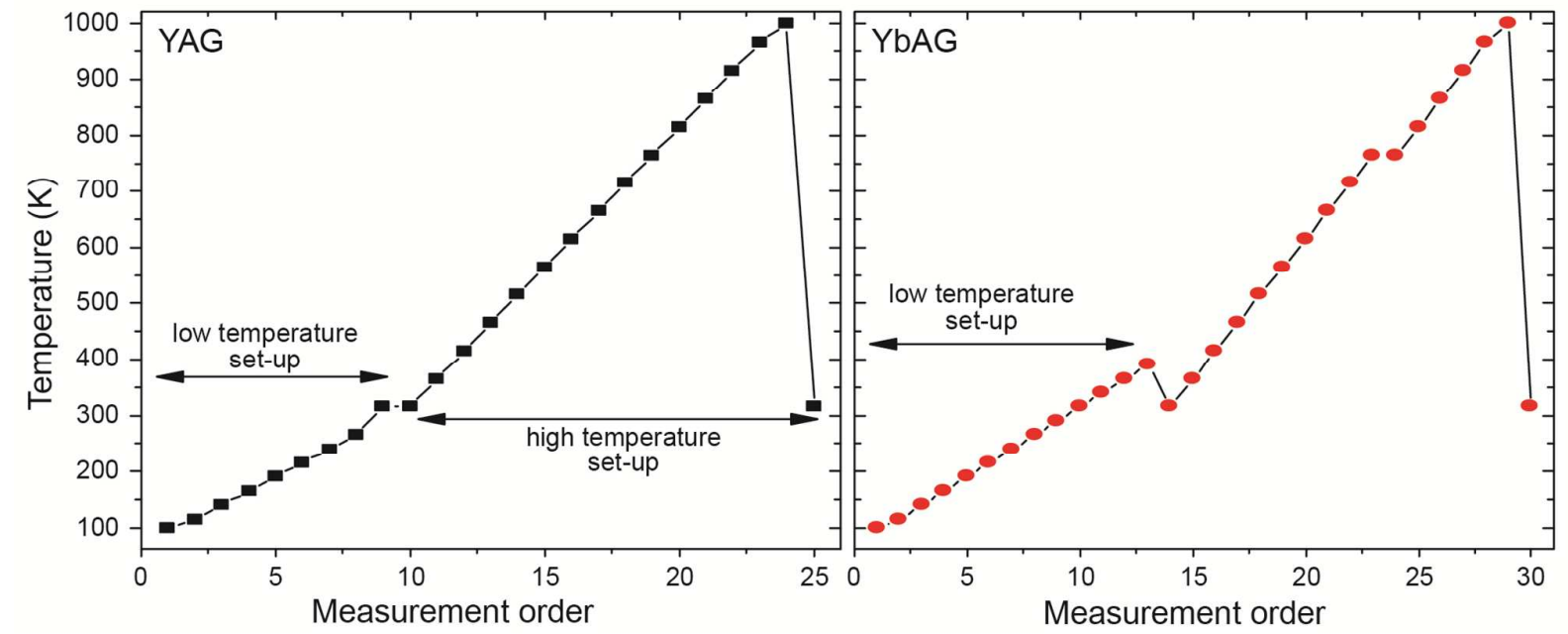

Figure S7: Unit cell as function of temperature extracted from Spring8 data for YAG and YbAG. 
Multi-temperature PXRD of YAG
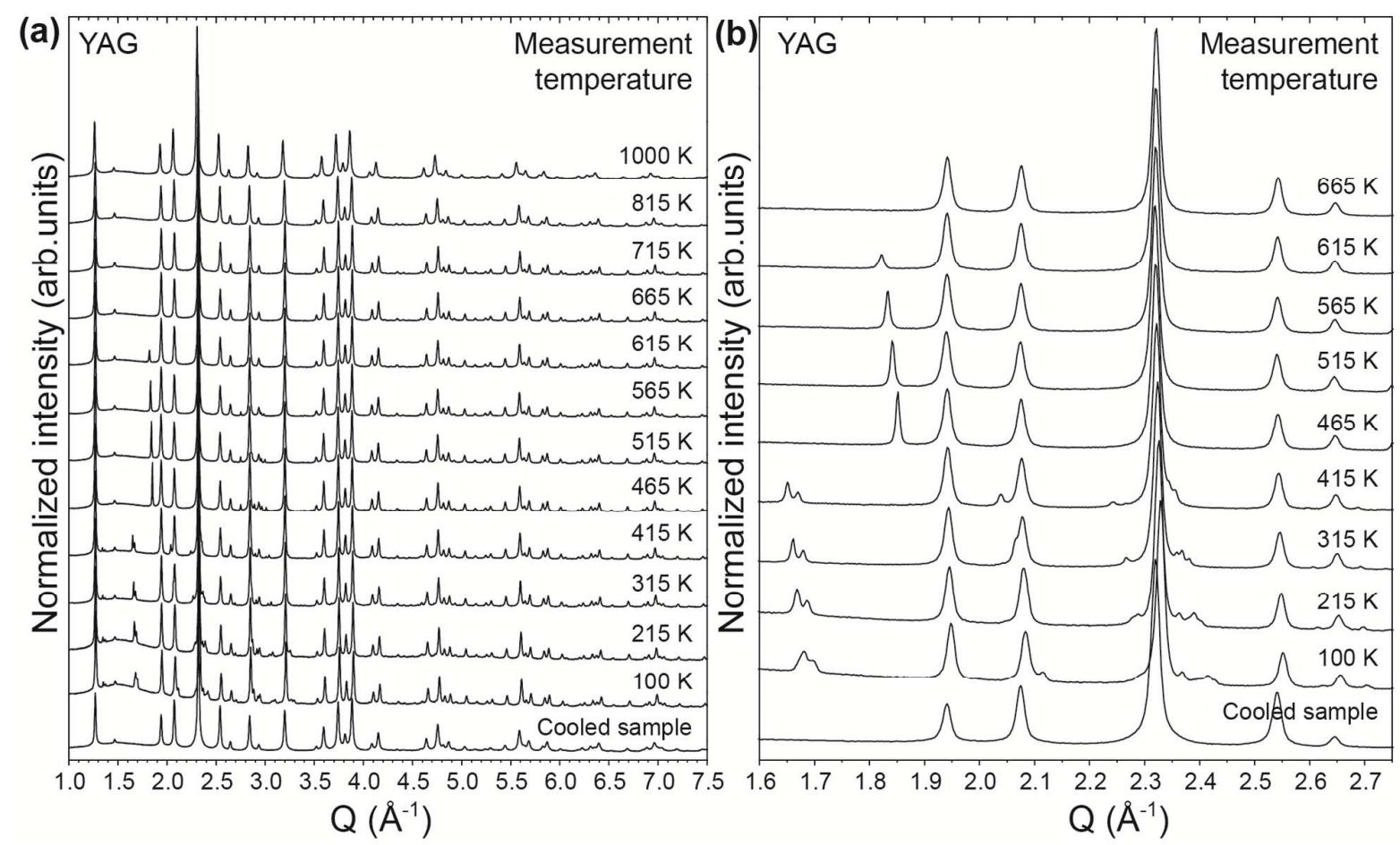

Figure S8: segment of the PXRD patterns for YAG obtained at SPring-8 and a zoom on the impurities phases, respectively, at selected representative temperatures. Cooled sample refers to the sample after it has been heated to $1000 \mathrm{~K}$ and cooled down to $315 \mathrm{~K}$ again.

\section{Strategy for the Rietveld refinement of the multi-temperature PXRD patterns obtained at SPring-8}

The positions of the oxygen atoms are fixed to the coordinates from the ICSD database for YAG (PDF no. 01-070-7794) and YbAG (PDF no.: 01-073-3186), respectively. Several different Rietveld refinement methods have been tried to figure out, which would give a realistic picture of the strange behavior of the lattice parameters. The best model is the one described in the paper. The following other models have been tested:

R.1. Refine the B-values for $\mathrm{Y}_{24 \mathrm{c}}, \mathrm{Al}_{24 \mathrm{~d}}, \mathrm{Al}_{16 \mathrm{a}}$ while keeping the occupancies at the stoichiometric values.

R.2. Refine the occupancies of $\mathrm{Y}, \mathrm{Al}_{24 \mathrm{~d}}$, and $\mathrm{Al}_{16 \mathrm{a}}$ while keeping the B-values constant at 1 .

R.3. Refine the occupancies such that the following reaction is fulfilled.

$$
\mathrm{Y}_{\mathrm{Y}}^{\mathrm{X}}+\left(\mathrm{Al}_{\mathrm{Al}}^{\mathrm{X}}\right)_{16 \mathrm{a}} \rightleftharpoons\left(\mathrm{Y}_{\mathrm{Al}}^{\mathrm{X}}\right)_{16 \mathrm{a}}+\mathrm{Al}_{\mathrm{Y}}^{\mathrm{X}}
$$

while keeping the B-values constant at 1 . 
R.4. Refinement of both the B-values and occupancies of the three atoms, but still fulfilling the anti-site reaction stated above. As there is a strong correlation between thermal displacement parameters (B-values) and occupancies special care has been taken that the right minimum is attained. Meaning that the occupancies has been refined in one cycle and afterwards held at its refined value while the B-values are refined. This has been done twice, before a simultaneously refinement of the occupancies and B-values have been done.

R.5. Almost the same as 4) but with the significant difference that the B-values are now constrained to have the same value for the three atoms, hence, only one B-value is refined in this refinement.

R.6. It was found that the anti-site reaction at some point was ineffective in describing the occupancy of sites correctly in R.4. As a consequence an additional parameter has been added in this refinement. When the anti-site reaction becomes ineffective is the occupancy of $\mathrm{Al}_{16 \mathrm{a}}$ freely refined. The $\mathrm{B}$-values are refined independently of each other.

R.7. Almost the same as 6) but the B-values for the different positions are now constrained to the same value; hence only one B-value is refined.

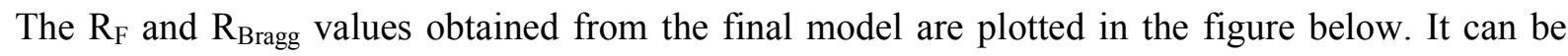
seen that the R-values for YAG are large owing to the undescribed impurity present in the sample. At $1000 \mathrm{~K}$, the R-values are similar for YbAG and YAG and around 2-3\%. Hence, the model is reliable describing the structure.

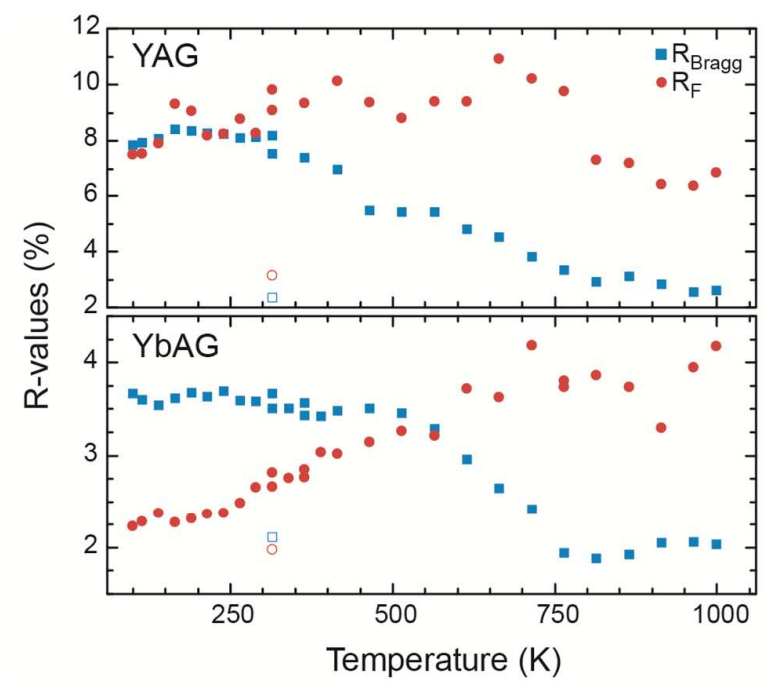

Figure S9 evolution of R-values as function of temperature. 